\title{
Couple characteristics and outcome of therapy in vaginismus
}

\author{
Thiloma Munasinghe ${ }^{1}$, Colvin Goonaratna ${ }^{2}$ and Padmal de Silva ${ }^{3}$
}

(Index words : Couple sex therapy, inappropriate surgery, non-consummation)

\section{Abstract}

Objective To describe couple characteristics and outcome of therapy in vaginismus.

Design A prospective before-after intervention descriptive study.

Setting Department of Physiology, Faculty of Medicine, Colombo, Sri Lanka.

Methods Fifty six couples with vaginismus, mostly self referrals and referrals from gynaecologists and general practitioners, were treated with a standard cognitive behaviour therapy protocol with before-after assessments of the degree of vaginismus and individual partner selfratings of the relationship and psychological status (GHQ-30).

Outcome measures Success at the end of the therapy was equated to the absence of or only mild vaginismus and, improvement in the couple relationship and psychological status scores.

Results Twenty seven (48\%) of the 52 (93\%) couples with non-consummation reported failure of coitus following previous non-surgical and surgical interventions. Love marriages $(70 \%)$, frequent attempts at sex $(75 \%, 3$ or 4 times/week) and sexual arousal (women $=86 \%$, men $=89 \%$ ) characterised couples. Ten men developed sexual problems, mostly erectile failure and premature ejaculation secondarily. Couple therapy enabled penetrative sex in $45(80.3 \%)$. The single prognostic indicator of outcome was the degree of vaginismus at first visit, those with mild and moderate vaginismus ( $77 \%$ ) being significantly more likely to establish coitus $(p<0.001)$ and complete the therapy $(p<0.001)$. The couple relationship improved marginally (women $p<0.01$, men $0.025<p>0.01$ ) but the psychological status remained unchanged. Dropouts [7] and referrals for psychiatric and marital counselling [4] failed to complete therapy.

Interpretation Couple sex therapy is effective in the management of vaginismus. Health professionals, especially gynaecologists and general practitioners, need to be aware of the problem and the satisfactory outcome of sex therapy.

\section{Introduction}

Non-organic vaginismus [1] is a psychosexual disorder in an otherwise normal woman, in whom attempted sexual intercourse triggers reflex spasm of the muscles surrounding the vagina, making penile penetration either impossible or painful. Data on the population prevalence of vaginismus is scarce [2], but clinic rates point to a higher incidence in conservative societies $[3,4]$ indicating that social and cultural factors are likely to influence prevalence. The many causes include relationship problems, anxiety, childhood sexual abuse, sexual ignorance, false beliefs about vaginal or penile size, fear of pregnancy and childbirth [3,5-7].

Primary vaginismus manifests as non-consummation or as primary infertility. The personal and social consequences adversely affect the quality of life of couples, especially in conservative societies. Avoidable causes of secondary vaginismus include vaginal trauma, infection or surgery as in poor obstetric practices at delivery [8].

Many uncontrolled trials and case series have established couple sex therapy as the preferred method of management of vaginismus[9]. Studies routinely exclude couples with negative prognostic indicators such as

${ }^{1}$ Medical Officer, The Central STD Clinic, National STD/AIDS Control Programme, Colombo; ${ }^{2}$ Professor of Physiology, Faculty of Medicine, Colombo and ${ }^{3}$ Senior Lecturer in Psychology, Institute of Psychiatry, Kings College, University of London. Correspondence: TM, Tel: +94 112 696444, e-mail: <tmunasinghe@ @otmail.com> (Competing interests: none declared). Received 1 December 2003 and accepted 15 January 2004. 
severe psychiatric morbidity, physical impediments, a lack of motivation and tendency for irregular attendance on the premise that they are unlikely to benefit from therapy [10]. Usually about $80 \%$ of couples achieve painless penetration after therapy, that is sustained in long term follow up, with improvement in the couple relationship and reduction of anxiety [10,11]. A misdiagnosed rigid imperforate hymen may result in unnecessary hymenectomy or hymenotomy [12] . Surgery often appears to worsen vaginismus, making subsequent sex therapy more difficult and less effective $[12,13]$.

Our experience of over a decade is that vaginismus causes much personal misery and tends to be managed inappropriately in Sri Lanka. Our objective was to describe vaginismus as a clinical entity and the outcome of a standard cognitive behaviour therapy protocol in its management.

\section{Patients and methods}

We obtained approval for the study from the Ethical Review Committee of the Faculty of Medicine, Colombo. Using a before-after study design we evaluated the outcome of a standard cognitive behavioural therapy protocol in a single group of 56 consenting couples with vaginismus, enrolled over a period of one year. Success at the end of the therapy was equated to the absence of or only a mild degree of vaginismus, and improvement in the partner relationship and current psychological status scores. A postal questionnaire checked sustainability of the outcome one year later. The outcome was determined at the last visit to ensure adequate end point data.

Using joint and separate interviews we collected data on socio-demographic characteristics, clinical symptoms and sexual history, and each partner self-assessed sexual knowledge and attitudes. The before-after rating of the couple relationship and current psychological status (GHQ-30 validated for Sri Lankan population [14]) was done separately by each partner. A single woman therapist (TM) managed the couples and the senior therapist (CG) carried out an independent assessment of outcome.

Therapy was provided according to a standard protocol. The initial vaginal examination confirmed the presence and severity of vaginospasm $[15,16]$ and the normality of the reproductive tract. The psychodynamics of vaginismus was discussed and couples reassured that the vagina was normal in size and that spasm could be overcome. The mainstays of treatment were the training in Kegel's excercises [17] to relax the pubo-coccygeal muscle and gain greater control of spasm and the stepwise dilation of the vagina using lubricated fingers (more natural than dilators). Coitus was temporarily banned. Sensual pleasure was enhanced and anxiety allayed by encouraging exploration of the non-erogenous and later the erogenous areas (breast and genitals) using touch.

Mutually acceptable non-coital sex practices were encouraged and the need to regularly practise the pelvic exercises and vaginal dilation at home was stressed. The fortnightly follow up visits monitored progress. When three fingers could be accommodated vaginally without pain and discomfort [18], couples were reintroduced to coitus starting with passive peno-vaginal containment in the woman-on-top position with gradual progression to coitus. Couples referred for psychiatric and marital counselling and surgery on request resumed sex therapy at their discretion.

\section{Data analysis}

Data was analysed using a PC and SPSS software package to determine factors associated with the outcome of the therapy, before-after change in the couple relationship and GHQ-30 scores. The Chi square test was used to determine significant associations.

\section{Results}

Couples from all provinces except the northern and the eastern sought therapy. The majority were self referrals (57\%), 23\% were referrals from gynaecologists, and $9 \%$ were from general practitioners.

Table 1 shows that non-consummation was due to vaginospasm occurring at every attempt at penetration. Pain, fear and a burning sensation in the vulva were common accompaniments. The mean duration of vaginismus (24.6 months, $\mathrm{SD}=21.9$ ) was less than the mean duration of marriage ( 26.2 months, $\mathrm{SD}=22.4$ ) as four couples reported secondary vaginismus.

\section{Table 1. Clinical features of vaginismus at first} consultation $(\mathrm{N}=56)$

Clinical features

Number

Symptoms

A tightening sensation in the lower abdomen, perineum, buttocks and upper thighs

Pain at attempted vaginal intercourse

Fear of vaginal intercourse

56

Burning /smarting sensation in the vulval area

Degree of vaginismus [16]

First degree (mild)

Second degree ( moderate)

Third degree (severe)

Fourth degree (refuses examination)

Nature

Global ( occurring every time )

Primary vaginismus

Secondary vaginismus
43

36

100.0

76.8

60.0

65.5

15

28

6

7

26.8

50.0

10.7

12.5

56

52

4
100.0 92.9 
The majority of couples were young (mean age in years: women $=29.8 \mathrm{SD}=5.4 ;$ men $=33, \mathrm{SD}=5.9$ ), well educated (men tertiary $52 \%$; women secondary $63 \%$ ) and had love marriage $(69.6 \%)$. Couples reported frequent attempts at sex ( 3 or 4 times/week $=75 \%$ ), and normal sexual responses (vaginal lubrication in women $=86 \%$; penile erection in men $=89 \%$ ). Sex was mainly non-coital and usually inter-crural (before marriage $=76.8 \%$, after marriage $=94.6 \%)$. Most women were anorgasmic $(66 \%)$. Notably, 12 men complained of developing multiple sexual problems after marriage, mostly erectile failure disorder [6], premature ejaculation [5] and waning of sexual desire [4]. About $18 \%$ of women reported other sexual dysfunctions, mostly lack of desire and aversion.

Partners were liberal in outlook but sexually ignorant (mean knowledge score: women $=2.4$, SD1.1; men $=$ 2.2, SD 1.1; good knowledge $\geq 3$ on a 5-point scale). Women were more likely to believe that the vagina was too small for penetration (women $44 \%$, men $17 \%$, $\mathrm{p}<0.05)$ and dirty $(44 \%)$, and feared childbirth $(33 \%)$ and pregnancy $(10 \%)$. The majority of partners though happily married were sexually dissatisfied. Women (32\%) more than men (17\%) seemed sensitive to the presence of a marital problem. The marital and sexual relationships were rated as personally important and partners faithful and responsible. Women were healthy $(96.4 \%)$, though a few reported minor gynaecological problems (7.2\%). Family backgrounds were stable $(98 \%)$ and of average religiosity (96\%). There was no history of sexual abuse in the woman partner. Table 2 shows that 27 couples (48\%) had failed to establish coitus despite various non-surgical and surgical measures, including Fenton's repair, the common method of managing vaginismus in Sri Lanka.

Table 2. Previous treatment for vaginismus $(\mathrm{N}=\mathbf{2 7})$

\begin{tabular}{lcr}
\hline Previous treatment & Number & $\%$ \\
\hline $\begin{array}{l}\text { Conservative management } \\
\quad \text { counselling, insertion of }\end{array}$ & & \\
$\quad$ graded dilators, fingers, & & \\
$\quad$ lubricants, drugs, etc) & 15 & 55.5 \\
Hypnosis & 1 & 3.7 \\
Fenton's repair & 5 & 18.5 \\
Hymenectomy & 2 & 7.4 \\
Dilation of vagina under & & \\
$\quad$ general anaesthesia & 3 & 11.1 \\
Manual rupture of the hymen & & \\
$\quad$ (without anaesthesia) & 1 & 3.7 \\
Total & $\mathbf{2 7}$ & $\mathbf{9 9 . 9}$ \\
\hline
\end{tabular}

Forty five couples $(80.3 \%)$ engaged in penetrative sex following therapy (Table 3 ) with marginal improvement in the relationship scores, greater for women $(\mathrm{p}<0.001)$ than men $(0.025<\mathrm{p}>0.01)$. No statistically significant change was noted in the current psychological status. The single prognostic indicator was the degree of vaginismus at the first visit, women with mild and moderate degrees of vaginismus being significantly more likely to resolve their problem $(\mathrm{p}<0.001)$ and complete the therapy $(\mathrm{p}<$ 0.001 ). The mean number of treatment sessions was 5.3 $(\mathrm{SD}=3.2$; range $1-18)$. There was $88.9 \%$ agreement on outcome of therapy in the nine couples who reported for blind assessment.

Table 3. Response to therapy for vaginismus ( $N=56$ )

\begin{tabular}{|c|c|c|c|c|c|}
\hline \multirow{2}{*}{$\begin{array}{l}\text { Degree of } \\
\text { vaginis- } \\
\text { mus [16] }\end{array}$} & $\begin{array}{l}\text { First } \\
\text { visit }\end{array}$ & & $\begin{array}{l}\text { Last } \\
\text { visit }\end{array}$ & & $\begin{array}{c}\% \\
\text { Resolution }\end{array}$ \\
\hline & & $\begin{array}{c}\text { No } \\
\text { vaginismus }\end{array}$ & $\begin{array}{c}\text { Mild } \\
\text { vaginismus }\end{array}$ & Total & \\
\hline Mild & 15 & 15 & - & 15 & $100(15 / 15)$ \\
\hline Moderate & 28 & 22 & 2 & 24 & $78.6(24 / 28)$ \\
\hline $\begin{array}{l}\text { Severe } \\
\text { Refuses }\end{array}$ & 6 & 1 & 2 & 3 & $50(3 / 6)$ \\
\hline examination & 7 & 2 & 1 & 3 & $42.9(3 / 7)$ \\
\hline
\end{tabular}

Yates corrected, $x^{2}=11.8149, \mathrm{df}=1, \mathrm{p}<0.001$

Referral to another service and dropout were mainly due to poor compliance, and marital and psychological stress. Among referrals, partners were older (mean age: women 32.8 years, men 36.5 years) and duration of vaginismus longer (mean: 42.3 months), but dropouts were similar (mean age: women 29.9 years, men 32.1 years; duration of vaginismus 27.9 months) to those completing the therapy.

\section{Discussion}

The paucity of services was reflected in the pattern of attendance. Affected couples sought relief from a range of health professionals. Love marriages probably sustained vaginismus because men were more reluctant to force penetration when women expressed fear or pain, and there was heavy dependence on non-coital sex (intercrural sex 94.6\%) for pleasure. Hence, the majority of couples were reasonably content despite failure to consummate. The cultural sensitivity of the issue and the dearth of services also probably led couples to endure the condition for nearly two years $(60.7 \%)$ until social pressure to have a child and fears of subfertility with advancing age finally drove them to seek help.

Following sex therapy, 45 out of 56 couples $(80.3 \%)$ with vaginismus consummated marriage. Overall, the couple relationship appeared to benefit from sex therapy. The greater improvement in self-ratings of women reflected their better response to sex therapy. There was no statistically significant change in the current psychological status of partners after the therapy, probably because most couples were content at the outset. The better partner relationship, lower psychopathology and higher motivation for treatment among couples with vaginismus has been associated with better outcome at therapy [11]. 
Culture related issues such as sexual ignorance and misconceptions appeared to be causally important in the absence of others factors, such as marital disharmony and anxiety.

\section{Conclusions}

Our data suggest that non-consummation is usually caused by mild or moderate vaginal spasm that is amenable to sex therapy. There is a need for informing health professionals, especially gynaecologists and general practitioners, that sex therapy is the preferred mode of managing vaginismus.

\section{References}

1. The International Classification of Diseases and related health problems ICD-10. 1992. WHO. Geneva.

2. O'Donohue W, Dopke CA, Swingen DN. Psychotherapy for female sexual dysfunction: a review. Clinical Psychology Review. 1997; 17: 537-66.

3. Weerakoon P. The aetiology of the unconsummated marriage. Proceedings of the Annual Sessions of the Sri Lanka Association of Community Medicine, Colombo. 1988.

4. Tugrul C, Kabacki E. Vaginismus and its correlates. Journal of Sexual and Marital Therapy 1997; 12: 23-34.

5. Barnes J. Primary vaginismus (Part 2): Aetiological factors. Irish Medical Journal 1986; 79: 62-50.

6. Butcher J. Female sexual problems. In Tomlinson J, ed. ABC of Sexual Health. London: BMJ Books, 1999: $19-22$.
7. Everaerd W, Laan E. Drug treatment for women's sexual disorders. Journal of Sex Research 2000; 37: 195-204.

8. Rafka N. Vaginismus and vaginal tears. American Journal of Obstetrics and Gynaecology 1988; 158: 1043-5.

9. McGuire H, Hawton K. Interventions for vaginismus (Review). The Cochrane Database of Systematic Review, 2003.

10. Hawton K. Treatment of sexual dysfunction by sex therapy and other approaches. British Journal of Psychiatry 1995; 167: 307-14.

11. Heiman JR, LoPiccolo J. Clinical outcome of sex therapy. Effects of daily V weekly treatment. Archives of General Psychiatry 1983; 40: 443-9.

12. Fordney DS. Dyspareunia and vaginismus. Clinical Obstetrics and Gynaecology 1978; 21: 205-21.

13. De Silva P. Psychological treatment of sexual problems. International Review of Psychiatry 1994; 6: 163-73.

14. Rodrigo EK (Personal communication). GHQ-30 validated for Sri Lankan population.

15. Bancroft J. Human Sexuality and its Problems. Edinburgh: Churchill Livingstone, 1989.

16. Lamont JA.Vaginismus. American Journal of Obstetrics and Gynaecology 1978; 131: 632-5.

17. Kegel AH. Sexual function of the pubo-coccygeus muscle. Western Journal of Surgery, Obstetrics and Gynaecology 1952; 60: 521-4.

18. Masters WH, Johnson VE Vaginismus. Human Sexual Inadequacy. 1st edn. Boston: Little, Brown and Company, 1970. 